\title{
From trash to treasure - Regenerative potential of placental tissue
}

\author{
D Hettiarachchi ${ }^{a}$
}

\begin{abstract}
The human placenta is a fetomaternal organ involved in nutrition, waste elimination and gas exchange between the mother and her developing fetus. In the recent years, it has gained popularity as an organ of immense regenerative potential. Thus, cells and tissues isolated from placentae, are being used for a multitude of clinical applications. As such there are 3 main entities that can be harvested from a term placenta which is usually discarded following delivery. Most research published in this area focuses on two main cell types mesenchymal stromal cells isolated from various parts of the placenta and epithelial cells isolated from amniotic membrane and the amniotic membrane by itself for its numerous biological properties. The two cell types show phenotypic plasticity and lineage specific differentiation potential. The aim of this review is to provide clinicians an insight to the regenerative capacity of cells and tissues of placental origin and to summarize their current clinical applications.
\end{abstract}

Sri Lanka Journal of Obstetrics and Gynaecology 2018; 40: 46-48

DOI: http://doi.org/10.4038/sljog.v40i2.7846

\section{Introduction}

The human placenta is the first organ to develop during embryogenesis. Studies conducted in mouse models have shown a correlation between its morphogenesis and the influence of signaling between placental trophoblast and embryonic cells reflecting the key role it plays as the interface between fetal and maternal environments ${ }^{1}$. In addition to its role in exchange of gases, nutrient and waste products it also acts as an important source of pregnancy-associated hormones and growth factors, and is involved in immune protection of the fetus. The two most important cell types in the placenta belong to either the trophoblastic lineage which provide the main structural and functional components required for the close contact between the maternal and fetal circulation and the decidual cells from maternal uterine tissue. The placenta represents a reservoir of progenitor, stem cells and epithelial cells that have been shown to differentiate into various cell types, including adipogenic, osteogenic, myogenic, hepatogenic, cardiac, pancreatic, endothelial, pulmonary and neurogenic lineages ${ }^{2}$. Thus, four regions of fetal placenta can be distinguished: amniotic epithelial, amniotic mesenchymal, chorionic mesenchymal, and chorionic trophoblastic. From these regions, the following cell populations are isolated: human amniotic epithelial cells (hAEC), human amniotic mesenchymal stromal cells (hAMSC), human chorionic mesenchymal stromal cells (hCMSC), and human chorionic trophoblastic cells (hCTC) ${ }^{3}$.

\section{Amniotic membrane}

The amnion or the amniotic membrane (AM) is a bi-layered membranous sac enclosing the amniotic cavity, which surrounds and protects the embryo. It is the first to develop among the three embryonic

a Lecturer, Human Genetics Unit, Faculty of Medicine, University of Colombo, Sri Lanka.

Correspondence: DH, e-mail: <dineshani@anat.cmb.ac.lk>

(D) http://orcid.org/0000-0002-1732-7339

Competing interest: The authors report no conflict of interest 


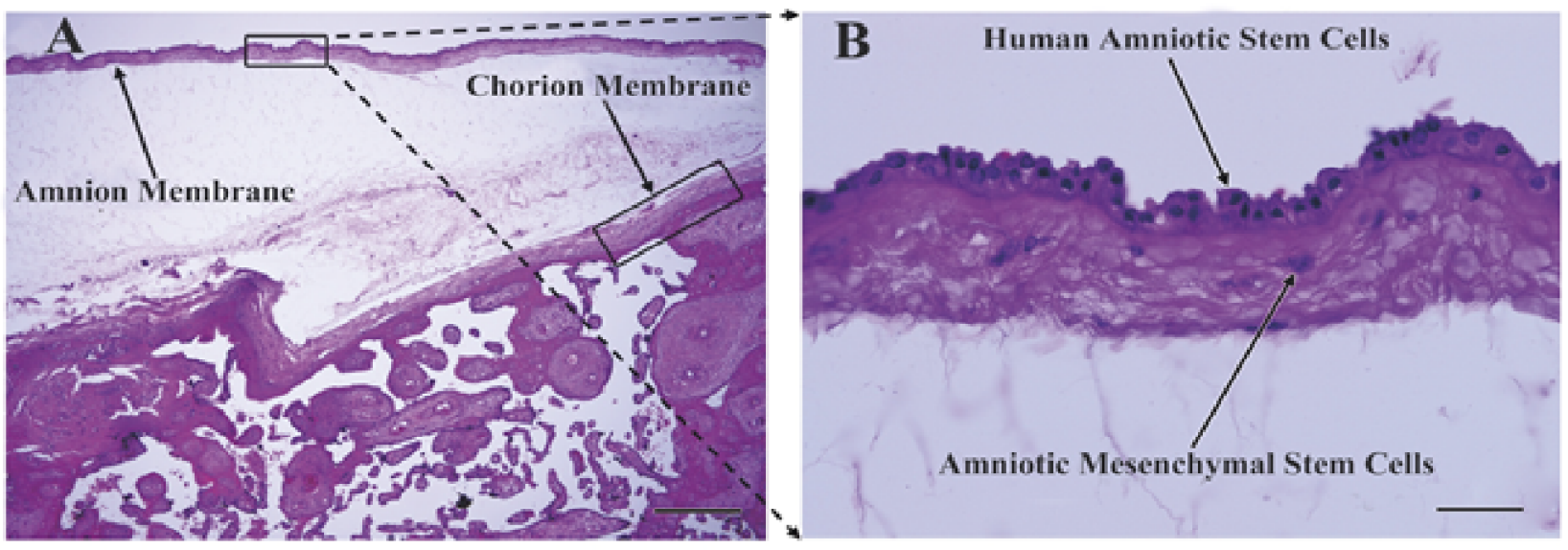

Figure 1. Cross section of the placenta. Haematoxylin and eosin (H\&E). A) Amniotic and chorionic membranes and the intermediate spongy layer are shown. B) Higher magnification of the amniotic membrane shows the single layer of flattened cuboidal cells resting on a basement membrane. Beneath this layer, thick avascular stromal layer containing spindle shaped amniotic mesenchymal cells is shown. A) scale bar; $500 \mu m$, B) scale bar; $50 \mu m$. Image reproduced with permission from Zarnani AH et al. (2014).

membranes (amnion, chorion and yolk sac) ${ }^{4}$. On microscopic examination, from the fetal surface inwards it consists of a single layer of cuboidal epithelium composed of human amniotic epithelial cells (hAECs) lying on a basement membrane (BM) and separated from the chorion by a loose avascular stromal matrix ${ }^{5,6}$ (figure 1). The epithelial layer is metabolically active; maintaining amniotic fluid homeostasis and secreting embryonic stem cell factors ${ }^{7}$. The BM improves epithelial cell migration and proliferation, strengthens cell adhesion, induces epithelial differentiation and prevents apoptosis. The stromal matrix secretes Transforming growth factor beta 1 (TGF$\beta 1)$ protease inhibitory factors, anti-angiogenic and anti-inflammatory factors which contribute to suppress fibroblast proliferation and differentiation, whilst inhibiting inflammation and neovascularization ${ }^{8}$.

Due to these unique properties, the AM has been used in wound care (leg ulcers, burns) and in ophthalmological applications which include treatment of neurotrophic corneal epithelial defects, shield ulcers, corneal abrasions, corneal ulcers, corneal burns, filamentary keratitis, dry eye and exposure keratopathy, recurrent corneal erosions, Salzmann's nodular degeneration, chemical and thermal burns and postinfectious keratitis ${ }^{6-8}$.

\section{Human amniotic epithelial cells (hAEC)}

Amniotic epithelial cells are generated from amnioblasts on the eighth day after fertilization and constitute the inner layer of the amnion. Ease of accessibly without any ethical constraints has made it an ideal noncontroversial source of primary cells that can be differentiated in a plethora of organ specific lineages, which makes these cells an ideal candidate for disease modeling and cellular replacement therapy. Owing to their epiblastic origin they show similarity to embryonic stem cells (ESC) by expressing pluripotent stem cell specific transcription factors such as stage specific embryonic antigen-3 (SSEA-3), SSEA-4 and tumor rejection antigen 1-60 (TRA1-60), TRA1-81, Octamerbinding protein 4 (Oct-4), Nanog and glucose-6phosphate dehydrogenase (G6PD) housekeeping gene and the potential to differentiate into the three germ cell layers ${ }^{9}$. Hence hAECs are a valuable source of functional cells that can be used in regenerative medicine and tissue engineering ${ }^{10}$. According to currently published data cell types of all three germ layers have been produced in vitro and there is strong evidence of neural, pancreatic, and hepatic differentiation of hAEC.

\section{Mesenchymal Stromal Cells from Amnion and Chorion: hAMSC and hCMSC}

These cells take origin from the extraembryonic mesoderm and exhibit properties of mesenchymal stem cells (MSC) with the ability to differentiate to mesodermal lineages such as adipose, connective tissue bone and cartilage ${ }^{11}$. Cell therapy with MSC has been used to treat a wide range of diseases and they are a promising cell resource for cell-based therapeutics because of their ability to self-renew and differentiate 
into specific functional cell types. The ClinicalTrials.gov registry currently lists more than hundred trials that are using exogenous MSC to treat a wide range of damaged, diseased or inflamed tissues ${ }^{12}$.

\section{Future direction}

Although there are many challenges to overcome in providing cell based therapies placental tissue remains a reservoir of progenitor stem cells with immense therapeutic potential in the future. Through investigation of its biology, discovery of their therapeutic mechanisms within animal models and testing their therapeutic potential within human trials we will hopefully make maximum use of this organ that is otherwise discarded.

\section{References}

1. Janet R, James C. Cross. "Placental development: lessons from mouse mutants". Nature Reviews Genetics 2.7 2001: 538.

2. Antoniadou, Eleni, David AL. "Placental stem cells". Best Practice \& Research Clinical Obstetrics and Gynaecology 2016; 31: 13-29.

3. Parolini, Ornella, et al. "Concise review: isolation and characterization of cells from human term placenta: outcome of the first international Workshop on Placenta Derived Stem Cells". Stem cells 26.2 2008: 300-11.

4. van Herendael B, Oberti C, Brosens I. Microanatomy of the human amniotic membranes. A light microscopic, transmission, and scanning electron microscopic study. American journal of obstetrics and gynecology. 1978; 131(8): 872-80.

5. Dobreva MP, Pereira PN, Deprest J, Zwijsen A.
On the origin of amniotic stem cells: of mice and men. Int J Dev Biol. 2010; 54(5): 761-77.

6. Chambers I, Colby D, Robertson M, Nichols J, Lee S, Tweedie S, Smith A. Functional expression cloning of Nanog, a pluripotency sustaining factor in embryonic stem cells. Cell. 2003; 113(5): 643-55.

7. Hao Y, Ma DH, Hwang DG, Kim WS, Zhang F. Identification of antiangiogenic and antiinflammatory proteins in human amniotic membrane. Cornea. 2000; 19(3): 348-52.

8. Kim JS, Kim JC, NA BK, Jeong JM, Song CY. Amniotic membrane patching promotes healing and inhibits proteinase activity on wound healing following acute corneal alkali burn. Experimental eye research. 2000; 70(3): 329-37.

9. Tabatabaei M, Mosaffa N, Nikoo S, Bozorgmehr M, Ghods R, Kazemnejad S, Rezania S, Keshavarzi B, Arefi S, Ramezani-Tehrani F, Mirzadegan E. Isolation and partial characterization of human amniotic epithelial cells: the effect of trypsin. Avicenna journal of medical biotechnology. 2013; 6(1): 10-20.

10. Miki T, Lehmann T, Cai H, Stolz DB, Strom SC. Stem cell characteristics of amniotic epithelial cells. Stem cells. 2005 ;23(10): 1549-59

11. Moore, KL, Persaud TVN, Torchia MG. "The Developing Human Clinically Oriented Embryology, 2008”. Philadelphia: Saunders.

12. Ankrum, James, and Jeffrey M. Karp. "Mesenchymal stem cell therapy: Two steps forward, one step back". Trends in molecular medicine 16.5 2010: 203-9. 